\title{
Compétences interactionnelles des tuteurs dans des échanges vidéographiques synchrones
}

\author{
Charlotte Dejean-Thircuir* — Nicolas Guichon** \\ Viorica Nicolaev*** \\ * Laboratoire Lidilem, Université de Grenoble \\ Domaine universitaire - BP 25, F-38040 Grenoble cedex 9 \\ Charlotte.dejean@u-grenoble3.fr \\ ** Laboratoire ICAR, Université de Lyon 2 \\ Centre de langues de Lyon 2, Bât. Filtre \\ 5 Avenue Pierre Mendès-France \\ F-69500 Bron \\ Nicolas.guichon@univ-lyon2.fr \\ *** Laboratoire ICAR, ENS-LSH \\ 15, parvis René Descartes, F-69366 Lyon cedex 07 \\ viorica.nicolaev@ens-lsh.fr
}

\begin{abstract}
RÉSUMÉ. Cet article s'intéresse aux dynamiques interactionnelles observables dans un dispositif didactique mettant en relation de futurs enseignants en formation en français langue étrangère avec des apprenants, par le biais de rencontres synchrones par visioconférence. Une analyse des interactions verbales permet d'identifier les conduites langagières des apprentis-tuteurs et s'attelle à déterminer dans quelle mesure celles-ci sont susceptibles d'influencer la mise en ouvre par les apprenants de certaines compétences de communication.

ABSTRACT. This article studies the interactional dynamics at work in a teaching set-up that puts future teachers of French as a foreign language in relation with distant learners via webbased videoconferencing. The analysis of the verbal interactions allows identifying whether the teacher trainees' verbal regulation deployed to manage the pedagogical interaction is conducive to the development of the learners' communication skills.

MOTS-CLÉS : formation à distance, communication vidéographique synchrone, apprentistuteurs, compétences interactionnelles.

KEYWORDS: distant language teaching, web-based videoconferencing, online teaching, teacher training, interaction competence.
\end{abstract}

DOI:10.3166/DS.8.377-393 @ Cned/Lavoisier 2010

D\&S - 8/2010. Enseignement des langues, pages 377 à 393 


\section{Introduction}

Les formations à distance en langue permettent la mise en œuvre de nouvelles formes d'échanges pédagogiques, non seulement grâce au dispositif technologique utilisé mais également grâce à des configurations sociopédagogiques inédites. On pense ainsi à des projets d'échanges pédagogiques tels que Cultura, Galanet ou encore le Français en première ligne (Develotte et Mangenot, dans ce numéro pour une présentation historique de ce projet). Ce dernier projet, sur lequel porte cette étude, donne l'opportunité à des apprentis-tuteurs en formation en Master 2 Didactique du français langue étrangère (désormais FLE) d'échanger plusieurs semaines de suite avec des apprenants étasuniens grâce à un dispositif vidéographique synchrone (Skype). Des objectifs ont été assignés à ces échanges par les partenaires institutionnels du projet, que l'on peut résumer ainsi : les apprentistuteurs doivent concevoir des tâches communicatives et les animer lors de la rencontre hebdomadaire, dans le but principal d'amener les apprenants à s'exprimer à l'oral. La visée essentiellement communicative de ces séances incite à se demander si les apprenants peuvent en effet exercer leur compétence de communication au cours des échanges. Nous formons l'hypothèse que cette possibilité est en partie liée à la médiation pédagogique des tuteurs et, spécifiquement, au mode de conduite de l'interaction par ces derniers.

Cette étude vise donc à mieux connaître les dynamiques interactionnelles dans un dispositif didactique nouveau pour les apprentis-tuteurs et pour les apprenants, afin de pouvoir formuler, à terme, des pistes en vue de la formation des tuteurs impliqués dans ce type de dispositif. Étant donné les spécificités à la fois techniques et sociopédagogiques de ce dernier, difficilement comparables avec des situations de classe, il paraît utile de pouvoir déterminer les conduites interactionnelles du tuteur susceptibles de favoriser l'implication des apprenants dans les échanges. Pour cela, nous adoptons une démarche caractéristique des approches interactionnistes en sciences du langage nous amenant à confronter une analyse quantitative et qualitative des échanges en ligne.

\section{Cadre théorique}

\section{Du développement des compétences chez les apprenants en ligne ...}

Depuis la fin des années 1990, de nombreux projets pédagogiques exploitant le potentiel des technologies numériques ont été mis en place pour permettre à des apprenants de développer leurs compétences langagières en échangeant en ligne avec des partenaires provenant d'institutions et de contextes linguistiques différents. S'appuyant tout d'abord sur des outils de communication médiatisée par ordinateur (désormais CMO) textuels et asynchrones comme le forum ou le courrier électronique, ces projets ont de plus en plus intégré des outils de CMO synchrones (par exemple le clavardage) et multimodaux. Ce n'est que récemment, grâce aux 
progrès technologiques réalisés d'abord dans le domaine de l'audioconférence puis de la visioconférence, que des projets recourant principalement à des outils synchrones ont pu voir le jour (cf. Hrastinski, 2008).

Quelle que soit la configuration temporelle et multimodale choisie, les recherches qui ont étudié les échanges entre pairs au sein de projets télécollaboratifs ont montré l'impact positif de ces dispositifs sur des aspects socio-affectifs liés à l'apprentissage d'une L2. Ainsi, il a été noté que ces échanges en ligne augmentaient la motivation des apprenants, diminuaient leur anxiété à manier la L2 et ménageaient des opportunités pour augmenter leur participation. (Beauvois, 1992; Kern, 1995 ; Sotillo, 2000 ; Warschauer, 1996). Un autre courant de recherche s'est penché sur le potentiel que ces échanges présentaient pour développer chez les apprenants des compétences interculturelles (Belz, 2003 ; Ware et Kramsch, 2005 ; O'Dowd, 2007 ; Audras et Chanier, 2008). Au-delà des aspects socio-affectifs et interculturels cruciaux pour l'apprentissage d'une L2, d'autres recherches ont exploré le potentiel de ces échanges en ligne pour le développement de certains aspects de la compétence langagière telles que les stratégies interactionnelles et discursives (Chun, 1994) ou la négociation du sens (Kost, 2008) déployées par les apprenants.

Dans le cadre de la présente étude, nous nous efforçons de comprendre le rôle du tuteur en ligne en étudiant d'une part, la capacité des apprenants « de s'engager dans des échanges communicatifs verbaux, de prendre position face à l'autre et de participer à la construction et à la régulation du discours » (Pekarek, 1999 : 10-11), d'autre part, leur capacité à construire un discours organisé et structuré pour « réaliser les différentes macro-fonctions (description, narration, argumentation, etc.) », comme cela est préconisé par le Cadre européen commun de référence (CECR, 2001 : 96). Ces compétences qui intègrent des dimensions interactionnelles et des dimensions plus textuelles sont appelées « discursives » par Pekarek (op. cit.) et «pragmatiques » par le CECR (2001 : 96-99). Nous adoptons pour notre part le terme «compétences pragmatiques», considérant que ce dernier englobe compétences interactionnelles et discursives.

\section{... au développement de compétences chez les enseignants en ligne}

Des projets originaux se sont multipliés depuis quelques années mettant en relation des apprenants de langue avec des enseignants de langue en formation, l'idée étant que tandis que les premiers développent des compétences langagières, les seconds mettent en place des compétences spécifiques à la pédagogie d'une langue en ligne (cf. Develotte, Guichon et Kern, 2007; Ware et Pérez-Cañado, 2007 ; Mangenot et Zourou, 2007). La mise en place de ces formations est née du constat qu'en même temps que les possibilités technologiques offertes par les outils de CMO synchrone se développent de nouvelles configurations d'enseignement des langues à distance se dessinent permettant de faire pratiquer la dimension orale de l'apprentissage de la L2, une dimension qui fut longtemps le parent pauvre de 
l'enseignement des langues à distance (Blake, 2008; Felix, 2002). Les pratiques pédagogiques qui en découlent, nécessitent que les enseignants puissent développer des compétences spécifiques lors de leur formation. Guichon (2009) s'est attelé à identifier les compétences nécessitées par cette configuration techno-pédagogique inédite :

- des compétences techno-sémio-pédagogiques, définies comme la capacité à utiliser les outils de communication à disposition et les modalités (texte, son, image, temps) qui leur sont propres de manière opportune ;

- des compétences de régulation socio-affective qui ont trait à la capacité de l'enseignant à établir et à maintenir, malgré la distance, un climat propice à la participation orale des apprenants et à leur prise de risque linguistique ;

- des compétences de médiation pédagogique. Elles concernent, d'une part, la capacité à concevoir des activités appropriées en amont de l'interaction afin d'optimiser l'engagement cognitif et émotionnel des apprenants dans l'échange. D'autre part, elles ont trait à la capacité de conduire l'interaction de façon à ce que les apprenants puissent développer leurs compétences pragmatiques. Précisons que cette capacité suppose une certaine « flexibilité communicative» qui renvoie à «la manière d'imposer ou non une planification [et] de laisser plus ou moins l'interaction être aussi dirigée par les élèves » (Bigot et Cicurel, $2005: 5$ ).

La présente recherche se focalise sur cette capacité interactionnelle des tuteurs à conduire localement les échanges avec des apprenants distants, et nous formons l'hypothèse qu'elle est pour partie liée aux compétences techno-sémio-pédagogiques propres à l'utilisation des outils de visioconférence.

\section{Corpus et méthodologie}

\section{Le projet "Français en $1^{\text {re ligne " }}$}

Le dispositif d'enseignement/apprentissage «Français en $1^{\text {re }}$ ligne », mis en place en 2002, a pour but de mettre en contact des étudiants en Master professionnel didactique du FLE qui apprennent à enseigner en ligne et des apprenants de langue française. Dans sa version initiale, cette formation s'appuyait sur un forum et privilégiait des échanges asynchrones (Develotte et Mangenot, 2007), mais depuis 2006, un autre dispositif recourant à la visioconférence poste à poste associée à un outil de clavardage a été mis en place pour former les apprentis enseignants aux particularités de l'enseignement en ligne synchrone (Develotte, Guichon et Kern, 2008 ; Guichon, 2009).

En 2007-2008, 10 apprentis-tuteurs de FLE, étudiants à 1'Université Lyon 2, et 17 apprenants de français (niveau B1), étudiants à l'UC Berkeley ont interagi par le biais d'un outil vidéographique synchrone (Skype) pendant sept séances d'environ quarante-cinq minutes. Ces séances s'inscrivaient dans le cadre pédagogique d'un cours de français de cinquante minutes qui avait lieu cinq fois par semaine pendant 
un semestre. Les apprentis-tuteurs français ont été chargés de la conception des tâches en fonction des contenus thématiques et linguistiques du programme suivi par les apprenants de Berkeley.

\section{Le corpus d'étude}

Notre étude s'appuie sur un corpus constitué de quatre sessions d'apprentissage en ligne (la $4^{\mathrm{e}}$ séance) et sur des entretiens semi-directifs. Ces derniers visaient à déterminer les perceptions des apprentis tuteurs vis-à-vis des outils à leur disposition, des relations construites avec les apprenants distants et de leur rôle pédagogique. Dans les quatre sessions en ligne, on retrouve quatre tuteurs et six apprenants selon les configurations présentées dans le tableau 1 qui détaille en outre certaines caractéristiques des tuteurs. On notera que seuls deux tuteurs sur les quatre (LET et MAR) pratiquaient la visioconférence (MSN) pour des usages sociaux avant cette formation.

\begin{tabular}{|c|c|c|c|c|}
\hline Tuteurs & Sexe & Age & $\begin{array}{c}\text { Expérience de la } \\
\text { visioconférence }\end{array}$ & Apprenants \\
\hline ANE & M & 29 & Non & LAN (M) \\
\hline JEA & M & 26 & Non & AND (F) \\
\hline LET & F & 31 & Usage social & DAN (M) et LAU (F) \\
\hline MAR & F & 23 & Usage social & PAU (M) et ARI (F) \\
\hline
\end{tabular}

Tableau 1. Caractéristiques des participants

Les quatre groupes ${ }^{1}$ (deux dyades et deux triades) ont travaillé à partir de la même séquence pédagogique dont le thème était «l'identité nationale». Elle comportait quatre activités prévues pour durer environ dix minutes chacune. La durée de la séance varie de 37 à 46 minutes suivant les groupes.

\section{La démarche méthodologique}

Les quatre sessions en ligne ont été enregistrées avec un logiciel de capture d'écran dynamique (Screen Video Recorder). Seules les données verbales ainsi que certaines données paraverbales ont été transcrites (cf. conventions de transcription en annexe). Cependant, pour l'analyse de certains passages, nous nous sommes

1. Pour guider le lecteur dans la découverte de l'exposé, nous avons nommé chaque séance de travail en accolant le numéro du groupe et le nom du tuteur (par exemple : groupe 1 - ANE). 
appuyés sur quelques indices non verbaux accessibles grâce aux enregistrements vidéo. Nous avons également tenu compte des tours de clavardage dans l'étude de ces séances.

Afin de mettre en relation les compétences pragmatiques des apprenants et les compétences interactionnelles des tuteurs, nous avons étudié plusieurs phénomènes interactionnels caractéristiques de l'analyse des interactions en classe de langue (Bouchard, 1984 ; de Nuchèze, 1984 ; Mehan, 1979; Van Lier, 1998, Pekarek, 1999) : la productivité des apprenants au cours de la séance, la façon dont sont gérés les thèmes dans l'interaction, les types d'intervention réalisés par les apprenants et la structuration des échanges. Une première exploration quantitative de certains phénomènes conversationnels est suivie d'une analyse qualitative des interactions verbales. Celle-ci est en outre croisée avec les discours des tuteurs issus des entretiens réalisés au terme de l'expérience, ces données étant susceptibles d'éclairer l'analyse des échanges.

\section{Quelques éclairages quantitatifs}

\section{Taux de participation}

Le taux de participation des interactants ${ }^{2}$ (cf. figure 1) est une première indication concernant la possibilité, pour les apprenants, de prendre la parole et d'exercer leurs compétences pragmatiques au cours des échanges.



Figure 1. Taux de participation des interactants

2. Nombre de mots produits par chaque interactant au cours de la session, rapporté au nombre total de mots produits durant celle-ci. 
Ces chiffres révèlent un écart important entre le taux de participation des apprenants lorsqu'ils sont seuls avec un tuteur ou en binôme. Cependant, on note qu'avec la même configuration de travail, des variations relativement importantes apparaissent d'un groupe à l'autre (40\% pour LAN dans le groupe 1 et $60 \%$ pour AND dans le groupe 2), ce qui tend à prouver que si cette variable est déterminante, elle n'est pas la seule à prendre en compte.

\section{Longueur des tours de parole des apprenants}

La longueur des interventions d'un apprenant constitue « un indice important de la prise de responsabilité de l'apprenant face à son activité discursive et d'une prise d'autonomie face au discours du [professeur] »(Pekarek, 1999: 67). La longueur moyenne des tours de parole des apprenants a été calculée en rapportant le nombre total de mots produits par les apprenants au nombre de tours de parole réalisés par chacun (cf. figure 2).

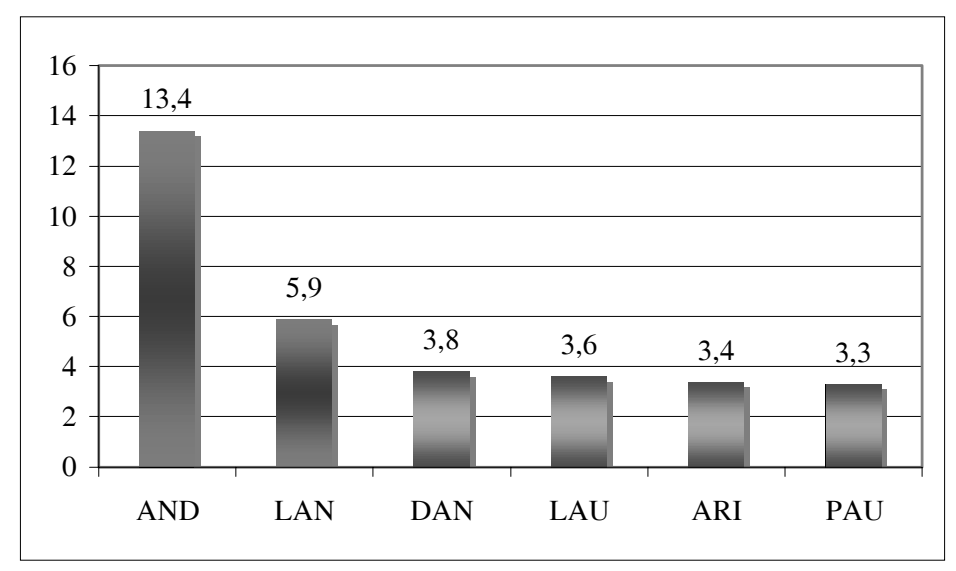

Figure 2. Longueur moyenne des tours de parole des apprenants

Les quatre apprenants qui travaillent selon la configuration 1 tuteur/2 apprenants présentent des moyennes quasi égales du point de vue de la longueur de leurs interventions. En revanche, l'apprenante AND qui interagit seule avec un tuteur produit des interventions nettement plus expansives (moyenne de 13,4 mots par intervention) que LAN qui travaille selon la même configuration (5,9 mots).

Quant au clavardage, il a été dans l'ensemble peu utilisé par les apprentis-tuteurs ou leurs apprenants, même s'il l'a été un peu plus dans le groupe 1. Nous avons relevé 42 interventions par clavardage du tuteur dans le groupe 1, 14 dans les groupes 2 et 3 et aucune dans le groupe 4 puisque l'outil n'a pas fonctionné. 


\section{Silences et chevauchements}

Pour analyser un échange didactique, le calcul des silences et des chevauchements (segments d'énoncés prononcés simultanément par au moins deux locuteurs) est un bon indicateur du rythme de l'interaction et peut témoigner de la capacité du tuteur à écouter et à laisser parler l'apprenant. Le tableau 2 quantifie la durée des silences inter-tours (silence entre deux tours de parole) et des silences intra-tours (silence à l'intérieur d'une prise de parole d'un participant) pour les apprenants et leur taux par rapport à la durée totale de la séance. Il présente également le nombre de chevauchements entre le tuteur (T) et le(s) apprenant(s) (A) dans les interactions étudiées. En effet, les chevauchements peuvent être révélateurs d'un certain dysfonctionnement de la gestion de l'alternance des tours et donc de l'interaction, notamment lorsqu'ils conduisent à une interruption du locuteur en train de s'exprimer. Dans le calcul effectué, les chevauchements intègrent tous les régulateurs (ou signaux d'écoute) qui ne sont pas a priori une gêne pour le déroulement de l'interaction, sauf s'ils sont en nombre excessif.

\begin{tabular}{|c|c|c|c|}
\cline { 2 - 4 } \multicolumn{1}{c|}{} & Silences inter-tours (\%) & $\begin{array}{c}\text { Silences intra-tours } \\
\text { des apprenants (\%) }\end{array}$ & $\begin{array}{c}\text { Nombre de } \\
\text { chevauchements } \\
\text { entre T et A }\end{array}$ \\
\hline $\begin{array}{c}\text { Groupe 1 } \\
\text { ANE/LAN }\end{array}$ & $6 \min 36 \sec (15 \%)$ & $2 \min 49 \sec (6 \%)$ & 53 \\
\hline $\begin{array}{c}\text { Groupe 2 } \\
\text { JEA/AND }\end{array}$ & $4 \min 41 \sec (10 \%)$ & $5 \min 34 \sec (12 \%)$ & 37 \\
\hline $\begin{array}{c}\text { Groupe 3 } \\
\text { LET/DAN, } \\
\text { LAU }\end{array}$ & $2 \min 16 \sec (4 \%)$ & $27 \sec (1 \%)$ & 174 \\
\hline $\begin{array}{c}\text { Groupe } 4 \\
\text { MAR/ARI, } \\
\text { PAU }\end{array}$ & $6 \min 44 \sec (18 \%)$ & $2 \min 32 \sec (7 \%)$ & 107 \\
\hline
\end{tabular}

Tableau 2. Silences et chevauchements dans les échanges

Alors que dans trois groupes sur quatre, les silences occupent en moyenne $22,6 \%$ de la durée de la séance de travail, ils n'occupent que $5 \%$ de celle-ci dans le groupe 3-LET. Par ailleurs, c'est également dans ce groupe que le nombre de 
chevauchements entre les énoncés des apprenants et ceux du tuteur sont les plus nombreux.

Si l'on prend en compte les différents paramètres analysés dans cette partie, les disparités observées entre les groupes, en particulier entre le groupe 2-JEA et le groupe 3-LET, constituent les premiers indices d'une variété de postures pédagogiques plus ou moins propices à la mise en œuvre de leurs compétences pragmatiques par les apprenants. L'analyse qualitative des échanges va permettre de montrer quelles sont les conduites langagières des tuteurs caractérisant ces postures.

\section{Analyse qualitative}

\section{Gestion du système d'alternance des tours}

Malgré le nombre limité de groupes observés, l'analyse de différents phénomènes liés à la gestion du système d'alternance des tours de parole confirme deux grandes tendances au niveau des conduites interactionnelles des tuteurs. On peut ainsi considérer que JEA (gr. 2) et LET (gr. 3) se situent aux deux pôles d'un continuum de postures tutorales plus ou moins favorables aux apprenants, illustrées ci-après. Notons que ANE (gr. 1) et MAR (gr. 4) se situent du côté de LET.

\section{Adopter une position d'écoute pour faire parler l'apprenante (groupe 2 -JEA)}

Dans le groupe 2-JEA, une proportion de silences relativement importante dans l'interaction, notamment à l'intérieur des tours de parole de l'apprenante (plus de 5 minutes pour moins de 3 minutes chez les autres), un petit nombre de chevauchements par rapport aux autres groupes et un taux de participation de l'apprenante supérieur à celui du tuteur tendent à montrer que ce dernier s'efforce de laisser parler celle-ci et de lui donner le temps de construire son discours. L'extrait suivant montre que même lorsque ses interventions sont ponctuées à la fois par de nombreuses hésitations et des pauses silencieuses (4), le tuteur n'intervient pas.

Extrait 1 (Gr. 2-JEA)

1. JEA et euh quel métier il ferait le français dans ton imagination

2. AND quel métier/

3. JEA oui métier

4. AND oui euh (1.5) les Français sont (3.4) les::: (1.0) les métiers c'est euh oui il fait les métiers comme (1.9) hum:: (3.4) comme un (3.4) euh couturier de vêtements (1.0) et comme (2.2) les euh vendeurs ou vendeuses de la nourriture dans la rue (1.6) et euh (1.2) euh (1.8) euh::(1.3) c'est euh (1.6) il travaille dans le métro (1.5) et aussi il (3.8) il fait la cuisinE et:: (4.4) oui c'est je ne peux pas pense aux autres 
Par ailleurs, ce tuteur a fréquemment recours à diverses procédures de relance pour inciter AND à poursuivre un énoncé inachevé ou à développer et justifier ses propos.

La position d'écoute de JEA et ses efforts pour faire parler l'apprenante sont évoqués par ce dernier au cours de l'entretien conduit à l'issue de la formation. Ces conduites sont à relier aux rôles qu'il attribue au tuteur et aux fonctions que ces séances de travail doivent remplir selon lui : « les faire discuter, les faire produire au maximum » (JEA). JEA poursuit ensuite sur les qualités que requiert ce rôle : «il faut être calme, patient ça c'est plus l'aspect technique, ne pas imposer, [...] c'est être discret, sinon c'est pas c'est pas intéressant, ne pas être présent, pas parler constamment». Les termes «patient», « discret», « ne pas être présent» sont particulièrement révélateurs de l'attitude que ce tuteur adopte au cours de l'interaction avec l'apprenante pour la « laisser parler ».

L'entretien montre en outre que la manière dont il gère le rythme de l'interaction est liée à une sensibilité particulière à ce phénomène : "si on était en face à face on leur laisserait du coup plus de temps je pense pour réfléchir [...] Je pourrais leur laisser du temps pour écrire je pourrais euh ce serait moins important de perdre du temps entre guillemets pour les laisser réfléchir pour les laisser imaginer enfin » (JEA)

Cette sensibilité constitue un atout pour ce tuteur qui est en mesure d'accepter une certaine lenteur du déroulement de l'interaction offrant ainsi à l'apprenante l'opportunité de construire des interventions longues et de mettre en œuvre certains aspects de ses compétences discursives. Néanmoins le dernier extrait révèle que les spécificités du dispositif (interaction à la fois médiatisée et d'une durée très limitée) ont pour effet de l'inciter à moins prendre le temps qu'il ne l'aurait fait en présentiel. On peut se demander si cette forme de pression temporelle, qu'a su malgré tout gérer ce tuteur, n'a pas constitué une difficulté importante pour les autres tuteurs.

\section{Limiter les pauses et occuper le terrain conversationnel (Groupe 3-LET)}

Au cours de la séance du groupe 3-LET, la durée des silences est très réduite par rapport à celle des autres groupes ( $5 \%$, par rapport à une moyenne d'environ $22 \%$ pour les autres). L'observation des échanges révèle que la tutrice semble intervenir de façon à éviter que le silence ne s'installe. Ainsi, après avoir formulé une consigne d'activité ou posé une question, elle a tendance à reformuler immédiatement son énoncé et/ou à questionner les apprenants pour vérifier qu'ils ont bien compris, sans leur laisser le temps de réagir. Elle intervient en outre fréquemment en chevauchement avec les énoncés des apprenants usant souvent de régulateurs pour ponctuer puis valider leurs prises de parole. Enfin, elle corrige beaucoup les apprenants soit pendant leur prise de parole soit immédiatement après, ce qui peut avoir pour effet d'interrompre leur tour de parole. 
L'occupation par la tutrice du terrain conversationnel (près de $70 \%$ du temps de parole total au cours de la séance) et la faible proportion de silences au cours de cette interaction par rapport aux autres groupes sont éclairés par certains de ses propos au cours de l'entretien :

«j'ai essayé d'être impliquée tout au long de l'interaction pour pour pour tenir euh comment dire euh pour que ce soit vivant en fait tout le long pour pas qui y ait de de d'impression que ça marche pas [...]» (LET)

On peut donc penser que pour celle-ci, les pauses, oralisées ou non, pourraient donner le sentiment d'une interaction qui ne "marche pas », ce qui la conduit à s'impliquer de façon excessive en limitant les possibilités de prise de parole et de construction par les apprenants d'un discours long et complexe. LET n'invoque pas le dispositif médiatisé pour justifier sa conduite mais on peut faire l'hypothèse que les silences, envisagés comme une rupture de contact malgré la vidéo, sont plus difficiles à appréhender pour le tuteur à distance qu'en face-à-face.

\section{Quelques remarques à propos du dispositif}

L'étude du système d'alternance des tours permet d'observer la gestion du rythme de l'interaction et l'ajustement des prises de parole entre les apprenants. Certains tuteurs (groupes 1,3,4) adoptent des conduites langagières qui limitent les possibilités d'expression et de déploiement de leurs compétences discursives par les apprenants, et qui semblent liées en partie à leur difficulté à prendre en compte les pauses et à les interpréter. Dans une interaction en face-à-face, le système de régulation qui permet le bon déroulement des échanges s'appuie en partie sur les indices mimo-gestuels émis par les participants. Grâce à la visioconférence, les tuteurs ont en partie accès à ces indices, ce qui permet d'identifier les réactions de l'apprenant et d'ajuster leurs interventions. Cependant, le dispositif technologique n'offre pas de véritable synchronie, ni au plan sonore ni du point de vue de l'émission du son et de l'image. Il amplifie certains phénomènes de délai dans la transmission des messages oraux et complique singulièrement la tâche de l'enseignant en ligne qui doit anticiper les décalages pour éviter de multiplier les chevauchements et se familiariser également avec l'écart entre parole et image. ANE (gr.1), qui affirme dans l'entretien s'être appuyé sur les informations non verbales que pouvait lui apporter la vidéo concernant les attentes de l'apprenant, a pourtant tendance à intervenir à la place de celui-ci en achevant ses énoncés par exemple, lorsque LAN rencontre des difficultés d'expression. Or, certains indices non verbaux émis par cet apprenant révèlent qu'il aurait parfois eu besoin de plus de temps pour construire son énoncé. Ainsi, l'absence de contact direct et la qualité relative de l'image associée aux décalages nécessitent une attention d'autant plus accrue à ces indices qu'ils sont moins accessibles qu'en face-à-face.

En outre, parmi les conduites ayant pour effet de limiter la parole des apprenants, il faut pointer les corrections en chevauchement ou immédiatement après les prises de parole de ces derniers. Pour effectuer les corrections, les tuteurs ont à leur 
disposition l'outil de clavardage leur permettant d'intervenir sans interrompre l'apprenant. Mais seul ANE (gr. 1) en fait un usage régulier, même si limité, qu'il justifie au cours de l'entretien par le fait de ne pas vouloir «casser la prise de parole» de l'apprenant. Ni JEA (gr. 2), ni LET (gr. 3), qui corrigent fréquemment oralement les apprenants, n'exploitent cette possibilité, peut-être en raison de la difficulté à maitriser la multimodalité qu'offre ce dispositif. Ceci renvoie à «la complexité de l'espace d'exposition discursive qui permet d'utiliser différentes modalités (orale, écrite, gestuelle) et à la polyfocalisation de l'attention (Jones, 2004) requise pour effectuer les différentes tâches » (Develotte et Mangenot, dans ce volume).

\section{Organisation des échanges et contrôle des thèmes}

Cette section porte sur la manière dont s'organise l'interaction au plan de la structuration des échanges ainsi que de la gestion des thèmes et vise à rendre compte de la place de chacun dans ce processus. Il convient tout d'abord de rappeler que ces sessions de travail entre un apprenti-tuteur et un ou deux apprenants sont fortement structurées autour des quatre tâches à réaliser selon le schéma pédagogique adopté. Le tuteur occupe une position centrale dans l'interaction car il initie l'essentiel des échanges en adressant à l'apprenant des requêtes, des demandes d'informations et des demandes de justification. Cependant, le degré de contrôle du tuteur sur les productions de l'apprenant peut varier fortement d'un groupe à l'autre.

Les réactions des apprenants peuvent être simplement ratifiées ou évaluées comme dans le schéma rituel des échanges scolaires correspondant à la structure ternaire IRE (incitation, réplique, évaluation; Mehan, 1979). Ce type d'échanges, toujours initiés par le tuteur, rend compte d'un faible degré de dialogicité ${ }^{3}$ dans les échanges, observable dans trois groupes sur quatre.

À l'inverse, dans le groupe 2-JEA, même si la majorité des échanges est déclenchée par des interventions du tuteur, le schéma IRE est moins fréquent que dans les autres groupes. Dans l'extrait suivant, JEA s'appuie sur les contenus apportés par AND pour conduire l'interaction, ce qui contribue à maintenir une cohérence discursive dans les échanges et à renforcer la co-construction de ces derniers. Prenant sa part de responsabilité dans la gestion des apports de contenu à l'interaction, l'apprenante exerce, de fait, sa compétence pragmatique.

Extrait 2 (Gr. 2 - JEA)

1. JEA (inaud.) d'accord (1.5) moi y a un truc que tu tu as dit c'est c'est sur les jours fériés tu as dit que c'est ce qui te faisait être [Américaine

3. «Renvoyant à l'occurrence d'échanges entre deux interlocuteurs qui dépassent trois tours de parole, la dialogicité décrit la mesure dans laquelle les E sont impliqués dans l'activité en cours plus que de façon ponctuelle » (Pekarek, 1999: 65). 
3. JEA c'est quoi ton jour férié préféré/

4. AND euh Noël (1.9) oui

5. JEA c'est Noël pourquoi/

Si trois tuteurs sur quatre ont quelques difficultés à s'appuyer sur les contenus apportés par les apprenants pour construire l'interaction à partir de ceux-là, et à s'éloigner de ce qui est prévu par le programme de la séquence, il est néanmoins deux situations au cours desquelles on peut observer ces phénomènes. Il s'agit d'abord des séquences d'ouverture qui précèdent le démarrage des activités, au cours desquels les participants sont dans des échanges plus informels portant sur leurs occupations, ce qui induit une certaine réciprocité dans le partage d'informations.

Ensuite, dans le cadre des activités prévues, l'apprenant devient producteur d'informations pour le tuteur, lorsqu'il est amené à parler de certains aspects de sa culture. Dans ces cas, il peut construire des interventions en faisant un usage plus libre de la langue et développer ainsi ses compétences pragmatiques.

Il est intéressant de souligner, en outre, que dans la configuration 1 tuteur/2 apprenants, c'est également lorsque les apprenants ont à s'exprimer sur leur environnement socioculturel qu'ils sont amenés à interagir entre eux, contrairement à ce qui se passe la plupart du temps. Généralement, lorsqu'un apprenant est en position de locuteur, il prend le tuteur comme interlocuteur et non l'autre apprenant. Même lors des activités censées les inciter à échanger entre eux, le tuteur redevient très rapidement l'interlocuteur privilégié. Dans l'extrait suivant, la discussion en cours porte sur les jours fériés et les apprenants expliquent ensemble à la tutrice ce qu'est «le jour du Président» aux Etats-Unis. La collaboration entre les deux apprenants pour construire un contenu informationnel pour la tutrice se manifeste à travers les phénomènes verbaux tels que l'achèvement interactif (6-7), la reformulation (8), l'apport d'informations complémentaires, ainsi que les phénomènes non verbaux (non notés dans la transcription) tels que l'échange de regards, les regards dirigés vers le partenaire, et l'orientation du buste dans sa direction.

Extrait 3 (Gr. 4 - MAR)

1. MAR le jour du Président

2. PAU oui

3. ARI oui

Sil (0.6)

4. MAR d'accord et c'est quel jour/

Sil (0.6) 
5. ARI hu:::m le

6. PAU euh (0.8) je pense que le

7. ARI le 18 février

8. PAU le deuxième lundi de cha- de chaque février

9. ARI oui

Ces observations révèlent que certains thèmes favorisant les comparaisons interculturelles sont davantage propices à la prise de parole de l'apprenant et à la construction d'interventions longues, qu'elles soient explicatives ou argumentatives. Par ailleurs, lorsqu'ils sont deux faces au tuteur, ces mêmes thèmes permettent aux apprenants de co-construire un discours, ce qui fait partie des compétences à développer oralement.

\section{Conclusion}

Cette étude montre tout d'abord que la configuration seul-à-seul avec le tuteur semble plus propice que la configuration qui met un tuteur en présence de deux apprenants en ce qui concerne le taux de participation de ces derniers (cf. figure 1). Quelle que soit la posture choisie par le tuteur, le temps disponible pour la prise de parole des apprenants est logiquement diminué et cela semble également jouer sur l'expansivité des énoncés qui sont plus longs quand il y a deux protagonistes plutôt que trois. Cependant, on a vu que la configuration à deux apprenants pouvait avoir d'autres avantages pour ces derniers, en leur permettant d'expérimenter parfois la co-construction d'un discours informationnel.

L'analyse des productions langagières des apprenants révèle la mise en œuvre limitée de leurs compétences pragmatiques (interventions courtes, rarement en position initiative dans l'échange, ne développant peu ou pas de discours argumentatifs et explicatifs) dans trois groupes sur les quatre observés. La mise en relation de ces résultats avec les différentes conduites interactionnelles des apprentis-tuteurs indique qu'il existe bien un lien entre ces dernières et le type de production des apprenants.

À la lumière de cette étude, nous pouvons résumer et préciser les compétences interactionnelles de médiation pédagogique que les tuteurs devraient s'efforcer de développer pour conduire des séances de tutorat par le biais de la visioconférence.

Il est à noter que certaines dimensions sont à relier à l'appropriation d'aspects techniques du dispositif. À ce propos, pour les quatre tuteurs observés la familiarité avec les outils de visioconférence pour des usages sociaux, ne semble pas avoir eu d'impact sur leur utilisation à des fins pédagogiques, puisque les tuteurs qui ont su gérer au mieux les spécificités du dispositif (JEA pour la prise en compte des pauses, ANE pour l'usage du clavardage) n'avaient pas eu d'expérience préalable de 
la visioconférence. Pour les tuteurs, il s'agit ainsi d'apprendre à recourir au clavardage quand cela est pertinent afin de minimiser le nombre d'interventions orales à visée corrective, sauf lorsque celles-ci sont sollicitées par les apprenants (cf. Guichon et Drissi, 2008). En outre, malgré l'absence de synchronie parfaite entre canal audio et canal visuel, les tuteurs doivent s'efforcer de prélever des indices non verbaux de type mimo-gestuel, notamment dans les moments de silences et de pauses silencieuses afin d'identifier les besoins de l'apprenant et d'ajuster les régulations. Par ailleurs, ils doivent apprendre à accompagner la prise de parole des apprenants par des régulateurs non verbaux (hochements, sourires, froncements) et limiter les régulateurs oraux potentiellement plus intrusifs. Il leur faut enfin accepter une certaine lenteur du rythme de l'interaction, et ménager des temps de silence et de pauses oralisées suffisamment longs pour permettre aux apprenants de comprendre les consignes et les questions, et de planifier leur discours.

D'autres compétences, en revanche, ne sont pas liées au dispositif technique, mais relèvent de compétences pédagogiques plus générales comme la capacité à relancer la parole ou la flexibilité communicative. Ainsi, apprendre à ajuster des compétences pédagogiques typiques de la conduite des interactions en classe de langue à cette situation de tutorat en ligne synchrone constitue un enjeu majeur de la formation des tuteurs, tandis que la visioconférence devient un outil de plus en plus stable et que son potentiel peut être efficacement exploité pour l'apprentissage d'une langue à distance.

\section{Bibliographie}

Audras I., Chanier T., « Observation de la construction d'une compétence interculturelle dans des groupes exolingues en ligne », Alsic, vol. 11, $\mathrm{n}^{\circ} 1$, 2008. http://alsic.revues.org/ index $865 . \mathrm{html}$.

Beauvois M. H., "Computer-assisted classroom discussion in the foreign language classroom: conversation in slow motion", Foreign Language Annals, vol. 25, n 5, 1992, p. 455-463.

Belz J., "Linguistic perspectives on the development of intercultural competence in telecollaboration", Language Learning \& Technology, vol. 7, n 2, 2003, p. 68-117.

Bigot V., Cicurel F., "Présentation. Les interactions en classe: contextes, ressources, enjeux ", Le français dans le monde. Recherches et applications, Les interactions en classe de langue, juillet 2005, Paris, CLE international, p. 4-6.

Blake R., "Distance learning for second and foreign language teaching", Van Deusen-Scholl N., Hornberger N.H., (Eds.), Encyclopaedia of language and education New York, Springer, vol. 4, 2008, p. 365-376.

Bouchard R., "Pour une méthodologie générale d'analyse des échanges verbaux en classe », Les échanges langagiers en classe de langue, 1984, Grenoble, P.U., Ellug, p. 73-110.

Chun D.M., "Using computer networking to facilitate the acquisition of interactive competence", System, vol. 22, n 1, 1994, p. 17-31. 
Conseil de l'Europe, Cadre européen commun de référence pour les langues. Apprendre, enseigner, évaluer, Paris, Didier, 2001.

Develotte C., Guichon N., Kern R., «"Allo Berkeley ? Ici Lyon... Vous nous voyez bien ?” Étude d'un dispositif de formation en ligne synchrone franco-américain à travers les discours de ses usagers », Alsic, vol. 11, n² 2, 2008, http://alsic.revues.org/index892.html

Develotte C., Mangenot F., « Discontinuités didactiques et langagières au sein d'un dispositif pédagogique en ligne », Glottopol, $\mathrm{n}^{\circ} 10,2007$, p. 127-144. http://www.univrouen.fr/dyalang/glottopol/

Felix U., "The web as a vehicle for constructivist approaches in language teaching", ReCALL, vol. 14, $\mathrm{n}^{\circ} 1,2002$, p. 2-16.

Garrison D.R., Anderson T., E-learning in the $21^{\text {st }}$ century. A framework for research and practice, New York, Routledge, 2003.

Guichon N., "Training future language teachers to develop online tutors' competence through reflective analysis", $\operatorname{Re} C A L L$, vol. $21, \mathrm{n}^{\circ}$ 2, 2009, p. 166-185.

Guichon N., Drissi S., «Tutorat de langue par visioconférence : comment former aux régulations pédagogiques ", Les Cahiers de l'ACEDLE, vol. 5, n 1, 2008, p. 185-217.

Guichon N., Nicolaev V., «Caractériser des tâches d'apprentissage et évaluer leur impact sur la production orale en L2 », Actes du colloque EPAL (Echanger pour apprendre en ligne : conception, instrumentation, interactions, multimodalité), Université Stendhal - Grenoble 3, 5-7 juin 2009.

Hrastinski S., “Asynchronous and Synchronous E-Learning”, EDUCAUSE Quarterly, vol. 31, $\mathrm{n}^{\circ} 4,2008$, p. 51-55.

Jacquinot G., « Apprivoiser la distance et supprime l'absence? Ou les défis de la formation à distance », Revue Française de Pédagogie, n 102, 1993, p. 55-67.

Jézégou A., « La distance en formation. Premier jalon pour une opérationnalisation de la théorie de la distance transactionnelle », Distances et savoirs, vol. 5, n 3, 2007, p.341-366.

Kern R., "Restructuring classroom interaction with networked computers: Effects on quantity and quality of language production", Modern Language Journal, vol. 79, 1995, p. 457-476.

Kost C. R., "Use of communication strategies in a synchronous CMC environment", Sieloff Magnan S. (ed.), Mediating discourse online, 2008, Amsterdam, John Benjamins, p. 153189.

Lamy M.-N., « Conversations multimodales : l'enseignement-apprentissage de l'oral à l'heure des écrans partagés ", Le Français dans le monde, Recherches et applications, Les échanges en ligne dans l'apprentissage et la formation, $\mathrm{n}^{\circ} 40,2008$, Paris, Clé international, p. 129-138.

Mangenot F., Zourou K., « Pratiques tutoriales correctives via Internet : le cas du français en première ligne », Apprentissage des langues et systèmes d'information et de communication, vol. 10, $\mathrm{n}^{\circ} 1,2007$, p. 65-99. http://alsic.org

Mehan H., Learning lessons. Social organization in the classeroom, Cambridge, MA/London, Harvard University Press, 1979. 
Nuchèze de, V. (1986), «Quelques propositions pour l'analyse pragmatique des échanges langagiers en situation didactique », Les échanges langagiers en classe de langue, Grenoble, P.U., Ellug, p. 41-72, 1984.

O’Dowd R. (ed.), Online Intercultural Exchange, Clevedon, Multilingual Matters, 2007.

Pekarek S., Leçons de conversation, Suisse, Editions universitaires de Fribourg, 1999.

Sotillo S., "Discourse functions and syntactic complexity in synchronous and asynchronous communication”, Language Learning \&Technology, vol. 4, n 1, 2000, p. 82-119.

Van Lier L., Interaction in the Language Curriculum - Awareness, Autonomy and Authenticity, London, Longman, 1996.

Ware P. D., Kramsch, C., "Toward an intercultural stance: Teaching German and English through telecollaboration”, Modern Language Journal, vol. 89, n² 2, 2005, p. 190-205.

Ware P.D., Pérez-Cañado M-L., "Grammar and feedback: Turning to language form in telecollaboration”, O'Dowd R., (Ed.) Online intercultural exchange: An introduction for foreign language teachers, 2007, Clevedon, England: Multilingual Matters.

Warschauer M., "Motivational aspects of using computers for writing and communication", Second Language Teaching\&Curriculum Center, 1996, http://nflrc.hawaii.edu/NetWorks /NW01?NW01.html

\section{Convention de transcription}

(2.0) pause égale ou supérieure à 0.5 secondes

$:$ allongement syllabique

/ montée intonative

[aaa le crochet indique le début du chevauchement

(rire) rire

(inaud.) segment inaudible 